\title{
CHANGE POINT TEST FOR DISPERSION PARAMETER BASED ON DISCRETELY OBSERVED SAMPLE FROM SDE MODELS
}

\author{
SANGyeOl LeE
}

\begin{abstract}
In this paper, we consider the cusum of squares test for the dispersion parameter in stochastic differential equation models. It is shown that the test has a limiting distribution of the sup of a Brownian bridge, unaffected by the drift parameter estimation. A simulation result is provided for illustration.
\end{abstract}

\section{Introduction}

Time series often experience structural changes governed by the change of financial policies and critical events. This phenomenon is prominent in most financial time series data with high volatility and is broadly known among practitioners. For instance, we can refer to Lee, Tokutsu, and Maekawa [9], who empirically demonstrated on the basis of CUSUM of squares test that most stock prices of Nikei 225 suffer from serious parameter changes when the underlying model of the data is assumed to be a $\operatorname{GARCH}(1,1)$ model. For a general review on the change point test in time series models other than GARCH models, we refer to Lee, $\mathrm{Ha}, \mathrm{Na}$, and $\mathrm{Na}[7]$, in which one can find various examples in discrete time series models such as random coefficient autoregressive models. Later, Lee, Nishiyama, and Yoshida [8] extended their method to continuous time stochastic processes. Recently, De Gregorio and Iacus [1] and Song and Lee [11] considered the change point test for the dispersion parameter in diffusion processes based on discretely observed sample. Also, see Iacus and Yoshida [2] for a multivariate version of this problem based on the quasi maximulm likelihood estimator. Compared to discrete time series models, the change point test in continuous time series models are not yet extensively studied. Hence, in this study, we consider the CUSUM of squares test constructed based on residuals for detecting the dispersion parameter change

Received January 5, 2010; Revised April 2, 2010.

2010 Mathematics Subject Classification. 62M86.

Key words and phrases. SDE models, diffusion process, discretely observed sample, residual based test, CUSUM of squares test. 
in stochastic differential equation (SDE) models. In particular, we consider the change point test for SDE models when the drift has unknown parameters.

The SDE models have long been popular in analyzing random phenomena occurring in various fields such as finance, engineering, physical and medical sciences. As a representative text, we can refer to Karatzas and Shreve [3] and Shiryayev [10]. Since the application of SDE models to real world is versatile, much attention has been paid to statistical inference for SDE models and many sophisticated methods have been designed by researchers and practitioners. For a general review, we refer to Kutoyants [5].

In Section 2, it is shown that under regularity conditions, the CUSUM of squares test based on residuals has a limiting distribution of the sup of a Brownian bridge. In Section 3, we carry out a simulation study.

\section{Main result}

Let us consider the stochastic differential equation:

$$
d X_{t}=a\left(X_{t} ; \theta\right) d t+b\left(X_{t} ; \sigma\right) d W_{t}, \quad X_{0}=x_{0}, t \geq 0,
$$

where $(\theta, \sigma)$ is a $p+q$-dimensional unknown parameter, $a, b$ are known real valued functions, and $W=\left\{W_{t} ; t \geq 0\right\}$ is a standard Brownian motion. Suppose that $X_{t_{i}}, t_{i}=i h_{n}, i=1, \ldots, n$, are observed, where $\left\{h_{n}\right\}$ is a sequence of positive real numbers such that $h_{n} \rightarrow 0$ and $n h_{n} \rightarrow \infty$. Based on this sample, one wishes to test the following hypotheses:

$$
H_{0}: \sigma \text { is constant over } i=1, \ldots, n \text { vs. } H_{1}: \operatorname{not} H_{0} .
$$

To this end, we consider the CUSUM of squares test based on residuals as in Lee, Tokutsu, and Maekawa [9]. We employ this test since it was proven very efficient for detecting change points in GARCH models. Compared to the test based on parameter estimators, it has merit of a high stability. For the same reason, we consider the CUSUM of squares test.

In what follows, we assume the following conditions:

(A1) There exist constants $C, m>0$ such that for any $(\theta, \sigma)$ and $x, y$,

$$
\begin{aligned}
|a(x ; \theta)-a(y ; \theta)|+|b(x ; \sigma)-b(y ; \sigma)| & \leq C|x-y|, \\
\sup _{\theta^{\prime} \in \mathcal{N}_{\theta}}\left\|\dot{a}\left(x ; \theta^{\prime}\right)\right\|+\sup _{\theta^{\prime} \in \mathcal{N}_{\sigma}}\left\|\dot{b}\left(x ; \sigma^{\prime}\right)\right\| & \leq C\left(1+|x|^{m}\right),
\end{aligned}
$$

where $\dot{a}=\partial a / \partial \theta, \dot{b}=\partial b / \partial \sigma$ and $\mathcal{N}_{\theta}$ and $\mathcal{N}_{\sigma}$ are open neighborhoods of $\theta$ and $\sigma$.

(A2) $\inf _{x, \sigma} b(x ; \sigma)>0$.

(A3) Under $H_{0}, \sup _{t} E\left|X_{t}\right|^{\gamma}<\infty$ for all $\gamma>0$.

(A4) Under $H_{0}$, there exists an estimator $\hat{\theta}_{n}$ of $\theta$ and $\hat{\sigma}_{n}$ of $\sigma$, such that $\left(n h_{n}\right)^{1 / 2}\left(\hat{\theta}_{n}-\theta\right)=O_{P}(1)$ and $n^{1 / 2}\left(\hat{\sigma}_{n}-\sigma\right)=O_{P}(1)$.

(A5) $n h_{n}^{2} \rightarrow 0$ as $n \rightarrow \infty$.

Sufficient conditions for (A4) can be found in Kessler [4]. 
By using the Euler approximation, we can naturally define the residuals as

$$
\hat{\eta}_{i}=\left\{X_{t_{i}}-X_{t_{i-1}}-h_{n} a\left(X_{t_{i-1}} ; \hat{\theta}_{n}\right)\right\} / b\left(X_{t_{i-1}} ; \hat{\sigma}_{n}\right) h_{n}^{1 / 2} .
$$

Then, we can obtain the following result.

Theorem 1. Assume that $(A 1)-(A 5)$ hold. Let

$$
T_{n}=\frac{1}{\sqrt{n} \hat{\tau}_{n}} \max _{1 \leq k \leq n}\left|\sum_{i=1}^{k} \hat{\eta}_{i}^{2}-\left(\frac{k}{n}\right) \sum_{i=1}^{n} \hat{\eta}_{i}^{2}\right|
$$

where $\hat{\tau}_{n}^{2}=\frac{1}{n} \sum_{i=1}^{n} \hat{\eta}_{i}{ }^{4}-\left(\frac{1}{n} \sum_{i=1}^{n} \hat{\eta}_{i}{ }^{2}\right)^{2}$. Then, under $H_{0}$, as $n \rightarrow \infty$,

$$
T_{n} \stackrel{d}{\rightarrow} \sup _{0 \leq u \leq 1}\left|W^{0}(u)\right|
$$

where $W^{0}$ is a Brownian bridge.

Remark. When $b$ is a constant function, we still have the above result for $h_{n}$ that satisfies $n h_{n}^{4} \rightarrow \infty$.

Proof. Put $\eta_{i}=\sigma\left(W_{t_{i}}-W_{t_{i-1}}\right) h_{n}^{-1 / 2}$. Then, we can express $\hat{\eta}_{i}=\eta_{i}+\Delta_{1 i}+$ $\Delta_{2 i}+\Delta_{3 i}$, where

$$
\begin{aligned}
\Delta_{1 i} & =\frac{\int_{t_{i-1}}^{t_{i}}\left\{a\left(X_{s} ; \theta\right)-a\left(X_{t_{i-1}} ; \theta\right)\right\} d s+\int_{t_{i-1}}^{t_{i}}\left\{b\left(X_{s} ; \sigma\right)-b\left(X_{t_{i-1}} ; \sigma\right)\right\} d W_{s}}{b\left(X_{t_{i-1}} ; \hat{\sigma}_{n}\right) h_{n}^{1 / 2}}, \\
\Delta_{2 i} & =\frac{\left\{a\left(X_{t_{i-1}} ; \theta\right)-a\left(X_{t_{i-1}} ; \hat{\theta}_{n}\right)\right\} h_{n}^{1 / 2}}{b\left(X_{t_{i-1}} ; \hat{\sigma}_{n}\right)}, \\
\Delta_{3 i} & =\left[b\left(X_{t_{i-1}} ; \sigma\right) / b\left(X_{t_{i-1}} ; \hat{\sigma}_{n}\right)-1\right] \eta_{i} .
\end{aligned}
$$

Since $E\left|\Delta_{1 i}^{*}\right|^{2}=O\left(h_{n}^{2}\right)$ (cf. Kessler [4]), where $\Delta_{1 i}^{*}$ denotes the numerator of $\Delta_{1 i}$, it can be easily seen that

$$
\frac{1}{\sqrt{n}} \sum_{i=1}^{n} \Delta_{1 i}^{2}=o_{P}(1)
$$

where we have used (A2) and (A5). Moreover, by (A1)-(A5), for all large $n$,

$$
\frac{1}{\sqrt{n}} \sum_{i=1}^{n} \Delta_{2 i}^{2} \leq C^{\prime} \sum_{i=1}^{n}\left(1+\left|X_{t_{i-1}}\right|^{m}\right)^{2}\left\|\hat{\theta}_{n}-\theta\right\|^{2} h_{n}=o_{P}(1)
$$

Similarly, we can obtain

$$
\frac{1}{\sqrt{n}} \sum_{i=1}^{n} \Delta_{3 i}^{2}=o_{P}(1)
$$

By using (2.3)-(2.5), one can eventually get

$$
\max _{1 \leq k \leq n} \frac{1}{\sqrt{n}} \sum_{i=1}^{n}\left|\hat{\eta}_{i}^{2}-\eta_{i}^{2}\right|=o_{P}(1)
$$

Since $\hat{\tau}_{n}^{2} \rightarrow \tau^{2}$ in probability, we obtain the theorem. 
Remark. The test cannot detect drift parameter changes as will be seen in our simulation study. Thus, the test is free from any drift changes. In fact, Lee and Guo [6] considered the constancy test for the dispersion parameter in the situation that $b(\cdot)$ is constant under the null hypothesis. They proposed a test, which is a hybrid of the Jarque-Bera's normality test and the Ljung-Box test, and figured out that the test is unaffected by the drift parameter estimation. The same phenomenon happens in our situation. From this, one can guess that instead of $T_{n}, T_{n}^{\prime}$ based on $\tilde{\eta}_{i}$ may be utilized, where

$$
\tilde{\eta}_{i}=\left(X_{t_{i}}-X_{t_{i-1}}\right) / b\left(X_{t_{i-1}} ; \hat{\sigma}_{n}\right) h_{n}^{1 / 2}
$$

and

$$
T_{n}^{\prime}=\frac{1}{\sqrt{n} \tilde{\tau}_{n}} \max _{1 \leq k \leq n}\left|\sum_{i=1}^{k} \tilde{\eta}_{i}^{2}-\left(\frac{k}{n}\right) \sum_{i=1}^{n} \tilde{\eta}_{i}^{2}\right|
$$

with $\tilde{\tau}_{n}^{2}=\frac{1}{n} \sum_{i=1}^{n} \tilde{\eta}_{i}^{4}-\left(\frac{1}{n} \sum_{i=1}^{n} \tilde{\eta}_{i}^{2}\right)^{2}$.

In the simulation study below, we will compare the efficiency of those two tests. In fact, it turns out that $T_{n}^{\prime}$ suffers from some size distortions and does not perform so well as $T_{n}$. This implies that to get an accurate result, one must employ the CUSUM of squares test that involves drift estimators.

\section{Simulation study}

In this section, we evaluate the performance of the CUSUM of squares test through a simulation study. In this study, we consider the CIR process:

$$
d X_{t}=\left(\alpha-\mu X_{t}\right) d t+\sigma\left(X_{t}\right)^{1 / 2} d W_{t}, t \geq 0 .
$$

Note that in this model, Condition (A2) is satisfied only when $2 \alpha>\sigma^{2}$.

The empirical sizes and powers are calculated as the rejection number of the null hypothesis ' $H_{0}$ : No changes occur in $\sigma$ ' out of 1000 repetitions. The empirical quantile value for $\sup _{0 \leq u \leq 1}\left|W^{0}(u)\right|$ can be founded in Table 1 of Lee et al. [7, page 784]. In each simulation, we generate the sample with $n=200,500,1000$, and employ the sampling time length $h_{n}=n^{-2 / 3}$. For the

TABLE 1. Empirical sizes of $T_{n}$ and $T_{n}^{\prime}$ with $h_{n}=n^{-2 / 3}$

\begin{tabular}{c|ccc}
\hline \hline & & $\alpha$ & \\
$n$ & 0.10 & 0.05 & 0.01 \\
\hline \hline 200 & 0.068 & 0.043 & 0.004 \\
& $(0.204)$ & $(0.147)$ & $(0.046)$ \\
500 & 0.080 & 0.037 & 0.011 \\
& $(0.167)$ & $(0.093)$ & $(0.029)$ \\
1000 & 0.084 & 0.044 & 0.007 \\
& $(0.143)$ & $(0.080)$ & $(0.018)$ \\
\hline \hline
\end{tabular}


TABLE 2. Empirical powers of $T_{n}$ and $T_{n}^{\prime}$ with $h_{n}=n^{-2 / 3}$

\begin{tabular}{c|cc}
\hline \hline \multirow{2}{*}{$n$} & $(0.5,1.0,0.1) \rightarrow(0.5,1.0,0.5)$ & $(0.5,1.0,0.1) \rightarrow(1.0,2.0,0.1)$ \\
\hline \hline 200 & 0.998 & 0.034 \\
& $(0.999)$ & $(0.134)$ \\
500 & 1.000 & 0.043 \\
& $(1.000)$ & $(0.086)$ \\
1000 & 1.000 & 0.053 \\
& $(1.000)$ & $(0.059)$ \\
\hline \hline
\end{tabular}

TABLE 3. Empirical sizes of $T_{n}$ and $T_{n}^{\prime \prime}$ with $h_{n}=n^{-2 / 3}$

\begin{tabular}{c|ccc}
\hline \hline & \multicolumn{3}{|c}{$\alpha$} \\
$n$ & 0.10 & 0.05 & 0.01 \\
\hline \hline 200 & 0.074 & 0.041 & 0.005 \\
& $(0.069)$ & $(0.034)$ & $(0.009)$ \\
500 & 0.098 & 0.044 & 0.006 \\
& $(0.094)$ & $(0.044)$ & $(0.007)$ \\
1000 & 0.099 & 0.046 & 0.009 \\
& $(0.097)$ & $(0.053)$ & $(0.009)$ \\
\hline \hline
\end{tabular}

TABLE 4. Empirical powers of $T_{n}$ and $T_{n}^{\prime \prime}$ with $h_{n}=n^{-2 / 3}$

\begin{tabular}{c|ccccc}
\hline \hline & \multicolumn{5}{|c}{$\sigma: 1.5 \rightarrow \sigma^{\prime}:$} \\
$n$ & 1.6 & 1.7 & 1.8 & 1.9 & 2.0 \\
\hline \hline 200 & 0.081 & 0.173 & 0.277 & 0.450 & 0.651 \\
& $(0.102)$ & $(0.229)$ & $(0.379)$ & $(0.573)$ & $(0.746)$ \\
500 & 0.124 & 0.342 & 0.727 & 0.898 & 0.985 \\
& $(0.159)$ & $(0.413)$ & $(0.776)$ & $(0.930)$ & $(0.991)$ \\
1000 & 0.242 & 0.691 & 0.951 & 1.000 & 1.000 \\
& $(0.265)$ & $(0.720)$ & $(0.963)$ & $(1.000)$ & $(1.000)$ \\
\hline \hline
\end{tabular}

empirical size, we consider the CIR process with $\alpha=0.5, \mu=0.2$ and $\sigma=0.5$. The empirical sizes are calculated at the nominal level $0.01,0.05$ and 0.1 , respectively. Table 1 shows that $T_{n}$ with $h_{n}=n^{-2 / 3}$ is much more stable than $T_{n}^{\prime}$ : the figures in the parentheses stand for the sizes for $T_{n}^{\prime}$.

In order to examine the power, we consider the two cases that the CIR process with $\alpha=0.5$ and $\mu=1.0, \sigma=0.1$ changes at $[0.5 n]$ to that with

Case 1: $(0.5,1.0,0.5)$;

Case 2: (1.0, 2.0, 0.1).

Note that in Case 1, only the dispersion part changes, and in Case 2, only the drift part changes. As anticipated, Table 2 indicates that the tests with 
$h=n^{-2 / 3}$ produce good powers in Case 1 while they do not detect the change in Case 2. Although $T_{n}^{\prime}$ has good powers, one has to keep in mind that it has severe size distortions. Hence, it can be concluded that $T_{n}$ is preferred to $T_{n}^{\prime}$.

In this simulation study, we also compare the performance of $T_{n}$ and $T_{n}^{\prime \prime}$, where $T_{n}^{\prime \prime}$ is the cusum test constructed based on parameter estimators. Here, we consider the situation that $\alpha=10$ and $\mu=1$ are known and $\sigma$ is unknown. As an estimator of $\sigma$, we employ the least squares estimator (LSE) for simplicity. The $T_{n}^{\prime \prime}$ is not exactly the same as the test in Song and Lee [11] in that their estimator is not the LSE and $n h_{n}^{2} \rightarrow 0$ is not satisfied. However, Table 3 exhibits that $T_{n}^{\prime \prime}$ does not have sever size distortions, and both the tests are stable. Further, Table 4 shows than $T_{n}^{\prime \prime}$ produces slightly better powers than $T_{n}$ when $\sigma$ is assumed to change from 1.5 to 1.6-2.0. From this result, we can reason that the CUSUM test as in Song and Lee [11] may outperform to a certain degree the CUSUM of squares test based on residuals, although the improvement would not be so remarkable. Past experience suggests that in general, the residual based CUSUM test is more convenient in implementation than the estimator based CUSUM test, since in the former case, there is no need to estimate the normalizing matrix that appears in the latter case.

Overall, this simulation study enables us to conclude that the CUSUM of squares test is a proper tool to detect the change of the dispersion parameter in diffusion processes. In fact, the test is desirable to design a method to detect changes for the drift part, since it is impossible for the CUSUM of squares test in this article to perform well. We leave this as a task of the future study.

Acknowledgements. I wish to thank the referee for his/her careful reading and helpful comments. This work was supported by the National Research Foundation of Korea (NRF) granting funded by the Korea government(MEST) (2009-0079414).

\section{References}

[1] A. De Gregorio and S. M. Iacus, Least squares volatility change point estimation for partially observed diffusion processes, Comm. Statist. Theory Methods 37 (2008), no. 13-15, 2342-2357.

[2] S. M. Iacus and N. Yoshida, Estimation for the change point of the volatility in a stochastic differential equation, Available at http://arxiv.org/abs/0906.3108.

[3] I. Karatzas and S. E. Shreve, Brownian Motions and Stochastic Calculus, Springer, New York, 1991.

[4] M. Kessler, Estimation of an ergodic diffusion from discrete observations, Scand. J. Statist. 24 (1997), no. 2, 211-229.

[5] Y. Kutoyants, Statistical Inference for Ergodic Diffusion Processes, Springer, New York, 2004.

[6] S. Lee and M. Guo, Test for dispersion constancy in SDE models, Submitted for Publication.

7] S. Lee, J. Ha, O. Na, and S. Na, The cusum test for parameter change in time series models, Scand. J. Statist. 30 (2003), no. 4, 781-796. 
[8] S. Lee, Y. Nishiyama, and N. Yoshida, Test for parameter change in diffusion processes by cusum statistics based on one-step estimators, Ann. Inst. Statist. Math. 58 (2006), no. 2, 211-222.

[9] S. Lee, I. Tokutsu, and K. Maekawa, The cusum test for parameter change in regression models with ARCH errors, J. Japan Statist. Soc. 34 (2004), no. 2, 173-188.

[10] A. N. Shiryayev, Essentials of Stochastic Finance, World Science, Singapore, 1999.

[11] J. Song and S. Lee, Test for parameter change in discretely observed diffusion processes, Stat. Inference Stoch. Process. 12 (2009), no. 2, 165-183.

Department of Statistics

Seoul National University

SEOUL 151-747, Korea

E-mail address: sylee@stats.snu.ac.kr 